\title{
Internet-based guided self-help versus group cognitive behavioral therapy for chronic tinnitus: a randomized controlled trial
}

Kristine Jasper, Cornelia Weise, Isabell Conrad, Gerhard Andersson, Wolfgang Hiller and Maria Kleinstaeuber

\section{Linköping University Post Print}

\section{Tweet}

N.B.: When citing this work, cite the original article.

Original Publication:

Kristine Jasper, Cornelia Weise, Isabell Conrad, Gerhard Andersson, Wolfgang Hiller and Maria Kleinstaeuber, Internet-based guided self-help versus group cognitive behavioral therapy for chronic tinnitus: a randomized controlled trial, 2014, Psychotherapy and Psychosomatics, (83), 4, 234-246.

http://dx.doi.org/10.1159/000360705

Copyright: Karger

http://www.karger.com/

Postprint available at: Linköping University Electronic Press

http://urn.kb.se/resolve?urn=urn:nbn:se:liu:diva-109400 
Internet-based Guided Self-help versus Cognitive Behavioral Group Therapy for Chronic Tinnitus: A Randomized Controlled Trial

Kristine Jasper $^{\mathrm{a}}$, MSc, Cornelia Weise ${ }^{\mathrm{b}, \mathrm{c}}, \mathrm{PhD}$, Isabell Conrad ${ }^{\mathrm{a}}, \mathrm{MSc}$, Gerhard Andersson ${ }^{\mathrm{c}, \mathrm{d}}$, $\mathrm{PhD}$, Wolfgang Hiller ${ }^{\mathrm{a}}, \mathrm{PhD}$, and Maria Kleinstäuber ${ }^{\mathrm{b}}, \mathrm{PhD}$

Author Note

a Department of Clinical Psychology and Psychotherapy, Johannes Gutenberg-University of Mainz, Mainz, Germany

b Department of Clinical Psychology and Psychotherapy, Philipps-University Marburg, Marburg, Germany

c Department of Behavioural Sciences and Learning, Linnaeus Centre HEAD, Swedish Institute for Disability Research, Linköping University, Linköping, Sweden

d Department of Clinical Neuroscience, Karolinska Institutet, Stockholm, Sweden

Corresponding Author: Maria Kleinstäuber, Philipps-University Marburg, Dept. of Clinical Psychology and Psychotherapy, Gutenbergstr. 18, D-35032 Marburg, Germany; e-mail: maria.kleinstaeuber@staff.uni-marburg.de; Phone: +49 (0)6421 2824051 (permanent address). 


\begin{abstract}
Background: The aim of this randomized controlled trial (RCT) was to investigate the effects of conventional cognitive behavioral face-to-face group therapy (GCBT) and an Internetdelivered guided self-help treatment (ICBT) on tinnitus distress.

Methods: A total of 128 adults with at least mild levels of chronic tinnitus distress were randomly assigned to GCBT $(n=43)$, ICBT $(n=41)$, or a web-based discussion forum that served as a control condition (DF; $n=44)$. Standardized self-report measures (Tinnitus Handicap Inventory, Mini-Tinnitus Questionnaire, Hospital Anxiety and Depression Scale, Insomnia Severity Index, Tinnitus Acceptance Questionnaire) were completed at the pre- and post-assessments and at the 6-month follow-up.

Results: Repeated measures ANOVAs revealed significant time x group interaction effects on the primary outcomes (Tinnitus Handicap Inventory, Mini-Tinnitus Questionnaire) in favor of both CBT interventions, compared to the DF at post-assessment $(0.56 \leq g \leq 0.93$; all $p s \leq$ $.001)$. There were no significant differences between GCBT and ICBT (all $p \mathrm{~s}>.05$ ), and treatment effects remained stable at the 6-month follow-up.

Conclusions: This study provides evidence that ICBT might be an equally effective alternative to conventional CBT in the management of chronic tinnitus. Despite encouraging results, further research is necessary to determine the actual potential of ICBT as a viable alternative to CBT, and under which circumstances it is effective.
\end{abstract}

Keywords: Internet-based therapy, cognitive behavioral group therapy, guided self-help, cognitive behavioral therapy, tinnitus 
Internet-based Guided Self-help versus Cognitive Behavioral Group Therapy for Chronic

Tinnitus: A Randomized Controlled Trial

Tinnitus is defined as the perception of sound without an external sound source [1]. Three to four percent of the adult population experience considerable distress from this condition [2]. Cognitive behavioral therapy (CBT) is one of the most researched tinnitus management interventions, but is rarely offered in clinical practice [3, 4]. The effectiveness of CBT on tinnitus distress has been demonstrated in reviews and meta-analyses [5-7].

Furthermore, a recent study by Cima et al. [8] revealed that a specialized CBT treatment for tinnitus had a high potential for widespread implementation in routine health care.

Over the last decade there has been a rise in CBT programs available on the Internet $[9,10]$. Internet-based CBT (ICBT) usually combines information delivery via the Internet (e.g., the presentation of therapeutic exercises) with therapist contact (e.g., via e-mail) and has shown promising effects for several psychosomatic or functional somatic syndromes in particular, such as chronic fatigue syndrome [11], chronic back pain [12], and irritable bowel syndrome $[13,14]$. The effectiveness of ICBT in the treatment of tinnitus has previously been tested in randomized controlled trials, where it has been contrasted with a wait list control group [15], a group-based CBT [16], an Internet-based acceptance and commitment treatment [17], and an information-only control program in an industrial setting [18]. Moreover, a non randomized clinical effectiveness study was carried out by Kaldo-Sandström et al. [19]. All of these trials reported positive effects on tinnitus distress.

Internet-delivered CBT is regarded as a viable alternative for people who are unable to access face-to-face CBT for different reasons, such as a long travelling time, not wanting the stigma of going to a therapist; or somatic obstacles like hearing impairment, or walking problems [20]. To answer the question of whether ICBT for tinnitus could be an equally effective alternative to face-to-face CBT, randomized controlled trials (RCT) that directly 
compare these two forms of therapy are needed [21]. There is currently only one small RCT in which ICBT for tinnitus has been compared with a face-to-face group CBT (GCBT) [16]. To enable maximum standardization Kaldo et al. [16] applied the same treatment manual, which was originally designed for use in ICBT, in both conditions. Both methods of treatment delivery led to similar significant improvements in tinnitus distress. However, there was no wait-list control group, and flaws in the implementation of the group treatment could have led to overestimation of the results in favor of ICBT [16]. In addition, the sample was relatively small ( $n=25$ and $n=26)$, resulting in low statistical power.

It therefore still remains unclear whether ICBT for tinnitus could be an equally effective alternative to conventional CBT. In order to enhance the external validity, we aimed to compare ICBT for tinnitus with a standard CBT program used in routine health care. To date, most of the well-tested CBT programs for tinnitus are delivered in a group format [6]. Moreover, similar to ICBT group treatments are potentially time efficient and cost-effective $[22,23]$, which suggests that both should be compared. Therefore, we decided to compare guided ICBT with a face-to-face group therapy. The GCBT treatment that was chosen as a comparison condition is regarded as a standard psychological tinnitus treatment and has been applied for many years in several psychotherapeutic outpatient and inpatient clinics. Accordingly, its efficacy has been confirmed in several studies [22, 24]. For the control condition, a monitored web-based discussion forum (DF) was used.

Based on previous findings concerning the efficacy of GCBT and ICBT that have been mentioned, we predicted that participation in both treatments would lead to a significantly higher reduction of tinnitus distress in comparison to the DF group. Furthermore, significantly greater improvements on secondary outcome measures of tinnitus acceptance, depression, anxiety, and sleeping disturbances were predicted for both treatments, in contrast to DF. Major benefits from participation in the DF group were not assumed, even though there are studies in which some improvements from active participation have been observed [25]. The 
effects of both treatments were expected to be stable at the 6-month follow-up. Moreover, it was predicted that both treatment conditions would not differ significantly regarding the reduction of tinnitus distress and general burden, according to secondary outcome measures at the post- and follow-up assessment. In addition, the association between treatment credibility, preference, or satisfaction with the intervention outcome was exploratively investigated.

\section{Method}

\section{Participants}

A total of 128 participants were treated between April 2010 and March 2011. They were recruited via a waiting list for tinnitus treatment at the University Outpatient Clinic for Psychotherapy in Mainz, the German Tinnitus Association, tinnitus self-help groups and public media. In addition, flyers were placed in pharmacies and the private practices of Ear, Nose and Throat (ENT) practitioners.

Criteria for study inclusion were (a) age of at least 18 years, (b) a score $\geq 18$ on the Tinnitus Handicap Inventory or a score $\geq 8$ on the Mini-Tinnitus Questionnaire [Mini-TQ; 26], (c) tinnitus duration of at least 6 months, (d) tinnitus as the primary problem, for example not as a consequence of morbus meniére, (e) consenting to be randomized, (f) Internet access, (g) willingness and ability to attend the weekly group sessions, (h) no anticipated absence of more than 2 weeks during the course of the study, (i) no CBT for tinnitus within the last 2 years, (j) no ongoing psychological tinnitus treatment, $(\mathrm{k})$ no major medical or psychiatric condition, and (1) no acute suicidality.

\section{Procedure and Study Design}

The study was designed as a randomized controlled trial (RCT). Participants were included through a three-stage selection procedure (pre-assessment, telephone interview, faceto-face interview). The Web-Based Screening Questionnaire for Common Mental Disorders 
[WSQ; 27] was used to screen for comorbid psychiatric conditions. Every case that exceeded the WSQ cut-off scores was further examined with the International Diagnostic Checklists for ICD-10 and DSM-IV [IDCL; 28]. Excluded participants were informed about other treatment options and had the opportunity to sign up for the wait-list for regular tinnitus treatment offered at the Outpatient Clinic. Included participants were randomized to one of the three conditions (ICBT, GCBT, DF). Following post-assessment, participants of the DF condition were randomly assigned to either ICBT, or to GCBT due to ethical concerns. Randomization was achieved by an online service which uses a pseudo-random number algorithm (www.randomization.com) and was conducted by an independent psychologist.

A-priori power calculations resulted in an estimation that a sample size of $n=34$ per group would be necessary to discover a medium main effect $(f=.25, \beta=.80, \alpha=.05)$ for each treatment. In order to detect a medium time $\mathrm{x}$ treatment interaction effect $(f=.25, \beta=.80, \alpha=$ .05 ) for the two CBT interventions (ICBT and GCBT), a total sample size of $n=34$ was required. Given an expected dropout rate of approximately $10 \%$, we decided that at least 120 participants would be needed.

All data were collected via the Internet and participants were assessed at the following intervals: during the initial selection phase (pre-assessment), directly after the last treatment week or the last week of the participation in the discussion forum (post-assessment), and 6 months after the end of the treatment (6-month follow-up). Participants had to sign an informed consent. The study was approved by the Ethics Committee of the Department of Psychology of the University of Mainz (Germany) and registered at www.clinicaltrials.gov (ID: NCT01205906).

\section{Treatments}

Both treatments lasted ten weeks and combined standard CBT techniques (e.g., focus exercises, cognitive restructuring) with relaxation exercises. It was highlighted that the aim of 
the program was not to reduce tinnitus loudness, but instead to reduce tinnitus distress. Both interventions were conducted by the same therapeutic team of Masters-level clinical psychologists, who were either certified CBT-therapists $(\mathrm{CW}, \mathrm{MK})$ or in the second year of their CBT training (IS, KJ). All therapists were experienced in GCBT for tinnitus. They received a specialized training for ICBT and continuous supervision to guarantee adherence to the protocols. ICBT participants were randomly allocated to a therapist. Each CBT group was conducted by one therapist. Tables 1 and 2 give an overview of the contents of both treatments.

--- Please, insert Tables 1 and 2 about here. ---

Internet-based cognitive behavior therapy (ICBT). The ICBT intervention was based on an adapted German version of the Swedish treatment manual developed by Kaldo and Andersson [29]. The ICBT consisted of 12 mandatory and 6 optional text modules, each covering a particular topic (see Table 1). Participants obtained access to the web-platform four days before the treatment started, in order to gain familiarity with the treatment rationale. Each module included general information, suggestions for exercising, worksheets, and solutions for common problems. All treatment weeks followed the same structure: (a) download of the text modules, (b) reading general information, and (c) exercising in daily life. Once a week patients could communicate with the therapist via a secured online messaging system. Therapists were instructed to try to dedicate a maximum of ten minutes per week/per patient for e-mail communication.

Cognitive-behavioral group therapy (GCBT). Treatment was strictly manualized and lasted for 10 consecutive weeks, consisting of a weekly 90 minutes session (see Table 2). Group sizes varied from five to twelve participants. Sharing experiences, discussing individual coping strategies, and demonstrating exercises, were important components of the treatment. To facilitate understanding and practice, participants were given handouts and encouraged to complete homework assignments. 
Web-based discussion forum (DF). As Dobie [30] pointed out in a review of RCTs on tinnitus treatments, it is likely that even nonspecific professional support and counseling would be helpful in the treatment of tinnitus. In order to control for nonspecific effects such as increased attention or empathy, a discussion forum was included as a control condition. In the DF a new discussion topic was presented every week. Participants were encouraged to discuss and to comment on each other's postings. The topics did not include any strategies to improve tinnitus distress but instead focused on individual experiences and attitudes concerning tinnitus (e.g., "Do you think that the topic 'tinnitus' is over- or underrepresented in public media?"). The forum was closely monitored to make sure postings were appropriate.

\section{Measures}

All data were assessed online as previous research has shown that self-report measures tend to transfer very well when administered via the Internet [e.g., 31].

Primary outcomes. Two self-report measures for assessing subjective tinnitus distress were used: the Tinnitus Handicap Inventory [THI; 32, 33] and the Mini-Tinnitus Questionnaire [Mini-TQ; 26, 34].The internal consistency of the current study was high with Cronbach's $\alpha=.92$ for the THI and Cronbach's $\alpha=.81$ for the Mini-TQ.

Secondary outcomes. For assessing secondary outcomes, the Hospital Anxiety and Depression Scale [HADS; 35, 36], the Insomnia Severity Index [ISI; 37, 38], and the Tinnitus Acceptance Questionnaire [TAQ; 39, 40], were used. The internal consistencies for the current study were high, with $\alpha=.83$ for the HADS anxiety scale, $\alpha=.89$ for the HADS depression scale, $\alpha=.87$ for the ISI and $\alpha=.86$ for the TAQ.

The Credibility/Expectancy Questionnaire [CEQ; 41] was administered before randomization to determine whether participants viewed the two treatments as equally credible. The authors reported good internal consistencies in various samples, but also 
difficulties in confirming the expected two factor solution (i.e., high correlations between the two factors, no simple structure). Given these findings and the high intercorrelation between the expectancy and credibility subscales in the current sample (for both treatments $r>.70, p<$ .001 ), we decided to use a sum score based on all six items. The internal consistencies for the current study were acceptable, with Cronbach's $\alpha=.68$ for the ICBT version and $\alpha=.66$ for the GCBT version. In addition, participants were asked to state their treatment preference (i.e., ICBT or GCBT). At post-assessment, participants were asked to rate their treatment satisfaction on a 5-point likert scale, ranging from very satisfied to very unsatisfied.

Finally, the total amount of time therapists devoted to the participants was assessed for each treatment (for GCBT: time spent for group sessions and for individual questions from patients prior to/after the sessions; ICBT: time to write the weekly feedback, to provide access to the text modules, and to handle additional questions).

\section{Data Analysis}

Statistical analyses were conducted using IBM SPSS Statistics 20. All analyses are based on the intention-to-treat paradigm (ITT). For ITT analysis, the multiple imputation procedure offered by IBM SPSS Statistics 20 was used to replace single missing values. The procedure produced five data sets using the monotone multiple imputation algorithm [42]. These five imputed data sets were then analyzed by using standard procedures used for complete data and then by combining the results across these analyses. Additionally, data were analyzed for completers only. If there were no differences between the results of the analyses based on the completer and the ITT analysis, results of the latter were reported.

In the first step, 2 (measurement: pre- vs. post-assessment) x 2 (treatment: ICBT or GCBT vs. DF) ANOVAs were conducted to investigate effects of each CBT intervention in comparison to the DF group, between pre- and post-assessment. In the second step, differences between the two active treatments at all three evaluation sessions were analyzed. 
Therefore 3 (measurement: pre- vs. post-assessment vs. 6-month follow-up) x 2 (treatment: ICBT vs. GCBT) ANOVAs were calculated. To determine the magnitude of within-group changes, effect sizes (ES) using Cohen's $d$ based on pooled standard deviations and the corresponding $95 \%$ confidence intervals, were calculated. If necessary, signs of ES were reversed so that positive values indicate favorable outcomes.

Hedges' g was calculated to quantify between-group treatment outcome differences, with positive values indicating a higher improvement of ICBT. Moreover, the Reliable Change Index [RCI; 43] was calculated for the primary outcome measures (i.e., THI and Mini-TQ) as an indicator of clinically significant improvement. The RCI was calculated by the formula $R C=\mathrm{x}_{2}-\mathrm{x}_{1} / \mathrm{S}_{\text {diff }}$, where $\mathrm{x}_{1}$ represents a subject's pre-test score and $\mathrm{x}_{2}$ its posttest score. $\mathrm{S}_{\text {diff }}$ is the standard error of the difference between the two test scores, calculated from the standard error of measurement $S_{\mathrm{E}}$ by the formula $S_{\mathrm{diff}}=\sqrt{ } 2 *\left(\mathrm{~S}_{\mathrm{E}}\right)^{2}$. For the calculation of $\mathrm{S}_{\mathrm{E}}$, the internal consistency (see Cronbach's $\alpha$ of THI and Mini-TQ under Measures) and the standard deviation of the sample at baseline (Table 3), was used.

\section{Results}

\section{Participant Characteristics}

Figure 1 summarizes the recruitment and flow of participants throughout the study. Of the 174 individuals assessed for eligibility, 46 participants were excluded, leaving a total sample of 128 to be randomized. Of these 128 participants, 7 were lost to post-test for various reasons such as they perceived the treatment as too extensive and stressful, lost interest, or were unavailable.

$$
\text { --- Please, insert Figure } 1 \text { about here --- }
$$

Demographic data and clinical characteristics of participants included in the study are presented in Table 3. With the exception of one participant who was of Asian origin, all participants were Caucasian. In the current sample, $91 \%$ of the participants reported a prior 
treatment for tinnitus. The most commonly stated previous treatments were medications stimulating blood flow (73\%), orthopedic treatment (42\%), and relaxation training (39\%). There were no significant baseline differences between the three groups concerning demographic data and clinical characteristics (all $p \mathrm{~s}>.05$ ).

--- Please, insert Table 3 about here ---

Means and standard deviations for all measures and assessments are presented in Table 4. One-way ANOVAs showed that there were no significant pre-treatment group differences for any of the outcome measures (all $p \mathrm{~s}>.05$ ). One-way ANOVAs and chi-square tests did not reveal any significant differences between completers and dropouts, either in terms of demographic data, clinical characteristics, or the baseline level of outcome measures (all $p \mathrm{~s}>.05)$.

--- Please, insert Table 4 about here ---

\section{Analysis of the Efficacy of Treatments vs. Control Condition}

Repeated measures ANOVAs comparing the ICBT followed by the GCBT intervention, respectively, with the control condition, resulted in significant group $\mathrm{x}$ time interactions (Table 5) in relation to the primary outcomes. Participants who received ICBT or GCBT showed significantly reduced tinnitus distress compared to participants in DF (Table 4). In addition, higher improvements for ICBT or GCBT on most of the secondary outcome variables were found. For the HADS-D, no significant time x group interaction could be detected for ICBT and GCBT (Table 5). However, a significant main effect of time was found for ICBT, $F(1,83)=12.00, p<.001$, and GCBT, $F(1,85)=13.24, p<.001$. Moreover, the main effect of group was not significant for either GCBT vs. DF, $F(1,85)=1.30, p=.257$, or for ICBT vs. DF, $F(1,83)=1.32, p=.255$. This result indicates that all three study groups improved significantly in regard to depressive symptoms, but there were no significant 
differences between the groups. Complete case analyses resulted in similar findings on all measures, such as the ITT analyses.

\section{Analysis of the Efficacy of ICBT vs. GCBT}

Repeated measures ANOVAs to compare ICBT with GCBT did not reveal any significant interaction effects, either in terms of pre- to post-assessment, pre-assessment to 6month follow-up, or in terms of post-assessment to 6-month follow-up(Table 5). Moreover, there were no significant main effects of group, $F(1,82)=0.01-1.45, p=.232-.929$. Significant main effects of time occurred for all outcome measures, $F(2,164)=14.93$ 108.63, all $p \mathrm{~s}<.001$, indicating that both treatments were similarly effective at reducing tinnitus distress or tinnitus-associated problems and that these effects remained stable at the 6month follow-up. The ITT and complete case analyses produced similar findings on all measures.

\section{Effect Sizes}

Concerning the within-group effect sizes, the ICBT $(0.39 \leq d \leq 0.96)$ and GCBT $(0.42$ $\leq d \leq 1.29$ ) interventions yielded predominantly medium to large effects, whereas only very small effects were obtained in the DF $(-0.02 \leq d \leq 0.26)$. For both treatment groups, the effect sizes were medium to large for the primary outcome measures (Table 4). Both interventions yielded the largest improvements on the Mini-TQ (ICBT: $d=0.96$; GCBT: $d=1.29$ ) and the smallest improvements on the HADS-D (ICBT: $d=0.39$; GCBT: $d=0.42$ ). The effect sizes remained stable at follow-up and were comparable on all outcome measures for the ICBT and the GCBT group (Table 4). Regarding between-group effect sizes (Table 5) which quantify the differences between the two treatments, the largest effects emerged for the Mini-TQ ( $g=$ $0.28)$ and the THI $(g=-0.15)$, and both favored GCBT. 


\section{Clinical Significance of Both Interventions}

According to the reliable change index [43], an improvement of at least 4 points in the Mini-TQ and 14 points in the THI was necessary to be classified as a therapy responder. We observed a clinically relevant improvement (i.e., therapy responders) based on the THI at the post-assessment for $41 \%$ of the ICBT participants and $50 \%$ of the GCBT participants. At the 6-month follow-up, we encountered a responder rate of $49 \%$ for ICBT and 51\% for GCBT. For the Mini-TQ (at the post-assessment), we obtained a rate of $52 \%$ therapy responders in the ICBT group, compared to $65 \%$ in the GCBT group. At the 6-month follow-up, the corresponding numbers were $56 \%$ for ICBT and $76 \%$ for GCBT. Chi-square tests failed to show any significant differences concerning the number of therapy responders in the two intervention groups (all $p \mathrm{~s}>.20$ ), with the exception of the 6-month follow-up. Here, regarding the improvement on the Mini-TQ significantly more responders were identified in the GCBT group, $\chi^{2}(1, N=84)=3.86, p=.049$.

\section{Treatment Credibility, Preference and Satisfaction}

Prior to randomization, participants rated their preferred treatment as well as treatment credibility, for each intervention. Over all patients, $46 \%$ preferred the GCBT, and $21 \%$ were in favor of Internet-based treatment. The remaining 33\% stated no preference. No significant differences between both treatment groups regarding their treatment preference were observed, $\chi^{2}(2, N=128)=0.54, p=.762$. According to the CEQ, treatment credibility/expectancy was rated significantly higher for GCBT $(M=36.43, S D=7.97)$ than for ICBT $(M=33.16, S D=9.24), t(126)=-4.01, p<.001$.

After completion of both interventions, participants rated their treatment satisfaction on a 5-point scale, ranging from very dissatisfied to very satisfied and also indicated whether they believed that the other treatment would have helped more. Results show that participants of GCBT $(M=4.00, S D=1.16)$, in comparison to those of ICBT $(M=3.38, S D=0.86)$, were 
more satisfied, $t(75)=-2.66, p=.010$.Moreover, both groups differed significantly in their ratings regarding the helpfulness of the two interventions, $\chi^{2}(2, N=78)=9.56, p=.008$.In the GCBT condition, 70\% rated their treatment (i.e., GCBT) as the most helpful intervention, only $8 \%$ thought the other treatment (i.e., ICBT) would have helped more, and $23 \%$ did not expect any differences in efficacy between the two treatments. In the ICBT group, only $38 \%$ considered their intervention (i.e., ICBT) as the most helpful one, $5 \%$ expected the other treatment (i.e., GCBT) to be more effective, and 57\% regarded both interventions to be equally helpful.

Next, we examined whether treatment credibility/expectancy and treatment satisfaction showed any relationships with the change scores of the primary outcome measures (i.e., pre- to post-assessment and pre- to follow-up assessment). No significant relationships between treatment credibility or treatment satisfaction and improvement in tinnitus distress were found (all $p s>.05$ ).

\section{Treatment Adherence}

The number of attended group sessions in the GCBT group $(M=7.33, S D=2.95)$ served as an indicator of treatment adherence. At the post-assessment, ICBT participants were asked which modules they had worked through. The number of completed mandatory modules $(M=9.05, S D=3.27)$ was chosen as a proxy of treatment adherence. Whereas for

GCBT no significant correlations were obtained, for ICBT the number of completed mandatory modules was significantly correlated with the change scores (pre- to postassessment) in regard to tinnitus distress (Mini-TQ), $r=.33, p=.040$, and sleeping disturbances (ISI), $r=.33, p=.038$.

To compare the actual treatment intensity of both interventions, we defined at least five attended group sessions or six mandatory modules as a 50\% participation, and more than eight group sessions or ten mandatory modules as $80 \%$ participation. Finally, the participation 
in ten group sessions or twelve mandatory modules indicated a full treatment. There were $28 \%$ of the GCBT participants and $24 \%$ of the ICBT participants who completed the whole treatment, whereas $61 \%$ of the GCBT participants and $86 \%$ of the ICBT participants only completed $80 \%$. Finally, in both intervention groups, $86 \%$ of the participants completed at least the half treatment. A chi-square test revealed that the differences between both

intervention groups regarding the actual treatment dose were not significant, $\chi^{2}(3, N=84)=$ $0.25, p=.970$

In order to examine whether treatment intensity was a crucial factor in our study, we conducted a separate analysis featuring only those participants who received a treatment dose of at least $80 \%$. Complete case analyses resulted in similar findings on all measures such as the ITT analyses.

\section{Therapist Time}

On average, ICBT therapists sent eleven messages $(S D=4.27)$ to each patient and received nine $(S D=5.06)$. They spent 13.76 minutes weekly per patient. The corresponding amount of time for the GCBT group was 11.22 minutes (90 min-session per week, 10 participants, one therapist).

\section{Discussion}

The present study suggests that ICBT and conventional GCBT may have comparable effects on tinnitus distress and associated problems. Furthermore, the discussion forum as an active control group, was found to consistently yield significantly lower improvements than both intervention groups, except on the HADS depression scale where all three study groups yielded similar effects. This result might be due to the low levels of depressive symptoms across all conditions. The 6-month follow-up assessment indicated that treatment gains of both interventions (ICBT and GCBT) did not differ and were sustained on all measures. In 
addition, comparing the distribution of patients attending at least $50 \%, 80 \%$, or $100 \%$ of the treatment modules did not reveal any significant differences between ICBT and GCBT, indicating that participants in both groups received comparable levels of treatment.

The treatment effects in this study are similarly high as those that have been reported in other trials. The GCBT yielded even higher within-group effect sizes (Cohen's $d=0.81-$ 1.29) regarding tinnitus distress, in comparison to an earlier trial (Cohen's $d=0.76-0.96$; [24]). The ICBT effects (Cohen's $d=0.71-0.96$ ) are similar to those reported in a previous study (Cohen's $d=0.73 ;[16])$ and somewhat higher than in a recent meta-analysis on selfhelp interventions on tinnitus (Cohen's $\mathrm{d}=0.48$; [44]). Moreover, the between-group effect sizes of the two treatments (i.e., ICBT or GCBT versus DF) are comparable to the effects reported in meta-analyses on tinnitus treatment $(g=0.44-0.67[5,6])$. In terms of clinically significant improvements, predominantly no significant differences between ICBT and GCBT were observed. The only exception occurred on the Mini-TQ at the follow-up assessment, where GCBT yielded a significantly higher rate of therapy responders. The percentage of clinically significant improvements was similar $[16,17]$, or somewhat higher $[15,19]$, than in previous trials. However, comparability is limited by the fact that we used the reliable change index, whereas most other studies used a 50\%-reduction of the mean scores on the primary outcome measure, as criterion.

The number of participants who completed the whole treatment (i.e., about 30\%) was somewhat lower than in previous studies $[15,19]$, where 50 to 60 percent of the participants were rated as fully treatment adherent. This difference might be due to our more conservative definition of treatment adherence, in which participants were counted as fully treatment adherent if they had worked through all mandatory modules. In the above mentioned studies $[15,29]$, only a general feedback for every treatment week was required for treatment adherence. This does not necessarily mean that the subjects worked through all required modules. 
An important feature of the current trial was the assessment of treatment credibility for ICBT and GCBT prior to randomization. In line with earlier research [16, 19], GCBT was rated as more credible than ICBT, but this did not influence treatment effects on tinnitus distress. In the current study, more than half of the participants preferred GCBT, whereas only about a fourth opted for ICBT. Following treatment, 70\% of the GCBT participants rated their own treatment as the most helpful, compared to $38 \%$ in the ICBT. Most ICBT participants rated both treatments as equally supportive, with only a few participants rating the other treatment as more helpful.

The high cost-effectiveness of ICBT due to time saving is often proposed as one advantage of ICBT, as compared to face-to-face therapy [e.g., 16, 45]. The therapist time for GCBT for tinnitus reported by Kaldo et al. [16] was more than twice as long as that for ICBT. The current study did not find such a big difference in relation to therapist time between GCBT and ICBT. In the trial by Kaldo et al. [16], seven participants attended 120-min group sessions, in contrast to the current trial where 10 participants attended 90 -min group sessions. Moreover, with 14 minutes per week of therapist time, the usual upper limit of 10 minutes in ICBT was therefore exceeded in the current trial. The combination of shorter group sessions with more participants per group in GCBT, as well as the longer therapeutic assistance in ICBT in the current study, might have contributed to the lack of difference in therapist time between the two interventions.

Some limitations of the current study need to be addressed and provide important venues for future research. First, data on the equivalence of ICBT and GCBT has to be interpreted with caution. The statistical power was high enough to detect a medium sized difference between both interventions. Both groups share common intervention techniques for tinnitus, therefore it is possible that only small differences exist between the two conditions, which might be more detectable in a larger sample. As this is the first study to directly 
compare ICBT with a standard GCBT treatment used in routine health care for chronic tinnitus, further research is needed.

Second, the generalizability of the results is limited for different reasons. It is questionable whether participants in the current RCT can be seen as representative of persons suffering from tinnitus in the general population. In line with previous studies on ICBT [e.g., 17, 46, 47], a high proportion of participants in the current trial were highly educated (nearly $50 \%$ had an academic degree). Internet access was an inclusion criterion and the prescreening assessment was administered online, which might have influenced the self-selection of the current sample. The possibility of a sampling bias might also be supported by the fact that a substantial proportion of participants on the waiting list for routine group therapy in the outpatient clinic declined participation in the study, as they were either unable to use the Internet or did not accept the randomization procedure. Even though Internet samples are usually not completely representative of the population due to there being more urban individuals with a higher education level, higher income, and who are more likely to have access to the Internet than less educated people living in rural areas [48, 49], they are not necessarily less representative than traditional samples in psychology [49]. A recent study revealed that the implications of a sampling bias in Internet treatments could be overestimated [50]. The authors demonstrated that although a higher education level was associated with consent to participate in an Internet treatment, no significant association with the treatment outcome was found.

A third limitation stems from the application of the HADS $[35,36]$ as a secondary outcome measure. In addition to the psychometrical problems of the HADS [51, 52], it only measures core symptoms of anxiety and depression. Tinnitus patients usually suffer from a wide spectrum of psychiatric symptoms, which might have been overlooked in the current study. A wider measure of psychiatric symptoms (e.g., Brief Symptom Inventory; [ 53]) 
would have been more appropriate to assess relevant aspects of tinnitus distress beyond depression and anxiety, such as somatization or interpersonal problems $[54,55]$.

Fourth, comparability of the two CBT conditions is limited for different reasons, such as the format of therapy (i.e., individual versus group therapy). Individual and group therapy are concurrent forms of therapy, which is reflected in differences regarding the therapeutic processes. Some therapeutic processes are unique for group therapy (e.g., group cohesion) [56] and, in addition, individual and group therapy tend to focus on different aspects. Group therapies usually focus on interpersonal and interactional processes, whereas intrapsychic aspects seem more important in individual therapy [57, 58]. Both formats of therapy are generally of equal effectiveness [59], but similar to ICBT, group treatments are potentially more time efficient and cost-effective [22, 23], which suggests that they should be compared. Yet, comparing ICBT with individual face-to-face CBT for tinnitus remains an important issue for future research. Another aspect which limits comparability is that the ICBT and face-to-face treatment were not based on the same treatment manual. However, our intention was to compare the group therapy in the form in which it is used in routine health care, with a treatment that has been optimized for web-based delivery and already positively evaluated. In this way we ensured that our results have a high external validity, but it may come at the expense of a somewhat lower internal validity. The comparability of the two interventions is also limited by the fact that we cannot fully exclude the possibility of flaws in the implementation of the group treatment, which might have led to an underestimation of the effects of GCBT. No formal measures of adherence to treatment protocol (i.e., video monitoring) were taken, but the GCBT therapists underwent weekly supervision. In addition, prior to treatment the participants were asked if they preferred ICBT or GCBT. The high preference toward group therapy might have led to an underestimation of the effects of the ICBT (e.g., due to disappointment after randomization to ICBT), but no significant correlations between treatment credibility and outcome were found. 
Fifth, a further limitation concerns the follow-up assessment. We cannot make precise statements concerning the stability of our effects for durations beyond six months. However, previous studies suggest that the results are long lasting $[16,24]$.

Furthermore, the sole reliance on self-report data represents a limitation of the study. Self assessments involve rather subjective judgments about individual experiences and behaviors, but some aspects of tinnitus are possibly only accessible via expert ratings (e.g., medical/psychological professionals). The combination of self-report data with more objective assessments due to clinical interviews (e.g., structural tinnitus interview [60,61]) and audiological data would have been more appropriate to contribute to the complexity of the tinnitus phenomenon $[60,62]$. Here, the domain of clinimetrics can be helpful in the construction of new scales that tap important aspects of tinnitus distress, which could be overlooked by traditional measurement instruments. A clinimetric approach typically involves patients and clinicians rating the importance of questionnaire items for the assessment of a clinical syndrome such as tinnitus distress. Only items that are rated as important are included in the final rating scales $[63,64]$.

A final limitation addresses our measure of treatment adherence. We assessed only the number of attended treatment modules, which gives no information about the extent of training the patients actually did. To gain a deeper understanding of what the patient actually does in ICBT, future studies should involve more intensive measures of adherence, such as completed homework assignments and the amount of time spent in practice.

Overall, the results of this RCT suggest that a substantial proportion of persons suffering from tinnitus distress might respond equally well to ICBT and GCBT. The preference of the majority of the sample for GCBT did not influence the treatment effects. The fact that one third of the participants stated no specific preference could be due to the increasing presence of the Internet in everyday life. This suggests that Internet-based treatments may be a welcome alternative for patients who cannot gain access to face-to-face 
interventions for a variety of reasons. Therefore, more research on the potential of ICBT as a comparable effective treatment option for tinnitus patients is needed. If the results of the current study are replicated, it might be a valuable future perspective to further implement Internet interventions into mental health care [65]. In particular, ICBT for tinnitus could be a promising approach within stepped care models $[22,66]$. This means that resource-efficient interventions such as ICBT would be tried first, whereas more intensive treatments would be reserved for times when a good outcome is not achieved (i.e. stepped care approach; [67]). The integration of ICBT into regular care within stepped care models could bridge the gap between the high demand for tinnitus treatments and the limited available supply. 


\section{References}

1 Lockwood AH, Salvi RJ, Burkard RF: Tinnitus. The New England Journal of Medicine 2002;347:904-910.

2 Davis A, El Refaie A: Epidemiology of tinnitus.; in Tyler RS (ed) Tinnitus handbook. San Diego, CA, Singular, 2000, pp 1-23.

3 Andersson G: Psychological aspects of tinnitus and the application of cognitivebehavioral therapy. Clin Psychol Rev 2002;22:977-990.

4 Gander PE, Hoare DJ, Collins L, Smith S, Hall DA: Tinnitus referral pathways within the National Health Service in England: a survey of their perceived effectiveness among audiology staff. BMC Health Serv Res 2011;11:162.

5 Andersson G, Lyttkens L: A meta-analytic review of psychological treatments for tinnitus. Br J Audiol 1999;33:201-210.

6 Hesser H, Weise C, Westin VZ, Andersson G: A systematic review and meta-analysis of randomized controlled trials of cognitive-behavioral therapy for tinnitus distress. Clin Psychol Rev 2011;31:545-553.

7 Weise C, Heinecke K, Rief W: Biofeedback-based behavioral treatment for chronic tinnitus: Results of a randomized controlled trial. J Consult Clin Psychol 2008;76:1046-1057. 8 Cima RFF, Maes IH, Joore MA, Scheyen DJWM, El Refaie A, Baguley DM, Anteunis LJC, van Breukelen GJP, Vlaeyen JWS: Specialised treatment based on cognitive behaviour therapy versus usual care for tinnitus: A randomised controlled trial. Lancet 2012;379:19511959.

9 Ritterband LM, Andersson G, Christensen HM, Carlbring P, Cuijpers P: Directions for the International Society for Research on Internet Interventions (ISRII). J Med Internet Res 2006;8:e23. 
10 Andersson G: Using the Internet to provide cognitive behaviour therapy. Behav Res Ther 2009;47:175-180.

11 Nijhof SL, Bleijenberg G, Uiterwaal CS, Kimpen JL, van de Putte EM: Effectiveness of internet-based cognitive behavioural treatment for adolescents with chronic fatigue syndrome (FITNET): a randomised controlled trial. Lancet 2012;379:1412-1418.

12 Carpenter KM, Stoner SA, Mundt JM, Stoelb B: An online self-help CBT intervention for chronic lower back pain. Clin J Pain 2012;28:14-22.

13 Hunt MG, Moshier S, Milonova M: Brief cognitive-behavioral internet therapy for irritable bowel syndrome. Behav Res Ther 2009;47:797-802.

14 Ljótsson B, Hedman E, Andersson E, Hesser H, Lindfors P, Hursti T, Rydh S, Rück C, Lindefors N, Andersson G: Internet-delivered exposure-based treatment vs. stress management for irritable bowel syndrome: a randomized trial. Am J Gastroenterol 2011;106:1481-1491.

15 Andersson G, Strömgren T, Ström L, Lyttkens L: Randomized controlled trial of internet-based cognitive behavior therapy for distress associated with tinnitus. Psychosom Med 2002;64:810-816.

16 Kaldo V, Levin S, Widarsson J, Buhrman M, Larsen HC, Andersson G: Internet versus group cognitive-behavioral treatment of distress associated with tinnitus: A randomized controlled trial. Behav Ther 2008;39:348-359.

17 Hesser H, Gustafsson T, Lundén C, Henrikson O, Fattahi K, Johnsson E, Westin VZ, Carlbring P, Mäki-Torkko E, Kaldo V, Andersson G: A randomized controlled trial of internet-delivered cognitive behavior therapy and acceptance and commitment therapy in the treatment of tinnitus. J Consult Clin Psychol 2012;80:649-661.

18 Abbott JA, Kaldo V, Klein B, Austin D, Hamilton C, Piterman L, Williams B, Andersson G: A cluster randomised trial of an internet-based intervention program for tinnitus distress in an industrial setting. Cogn Behav Ther 2009;38:162-173. 
19 Kaldo-Sandström V, Larsen HC, Andersson G: Internet-based cognitive-behavioral self-help treatment of tinnitus: clinical effectiveness and predictors of outcome. Am J Audiol 2004;13:185-192.

20 Cuijpers P, van Straten A, Andersson G: Internet-administered cognitive behavior therapy for health problems: a systematic review. J Behav Med 2008;31:169-177.

21 Cuijpers P, Donker T, van Straten A, Li J, Andersson G: Is guided self-help as effective as face-to-face psychotherapy for depression and anxiety disorders? A systematic review and meta-analysis of comparative outcome studies. Psychol Med 2010;40:1943-1957. 22 Kröner-Herwig B, Frenzel A, Fritsche G, Schilkowsky G, Esser G: The management of chronic tinnitus: Comparison of an outpatient cognitive-behavioral group training to minimal-contact interventions. J Psychosom Res 2003;54:381-389.

23 Kröner-Herwig B, Hebing G, van Rijn-Kalkmann U, Frenzel A, Schilkowsky G, Esser G: The management of chronic tinnitus--comparison of a cognitive-behavioural group training with yoga. J Psychosom Res 1995;39:153-165.

24 Hiller W, Haerkötter C: Does sound stimulation have additive effects on cognitivebehavioral treatment of chronic tinnitus? Behav Res Ther 2005;43:595-612.

25 Winzelberg AJ, Classen C, Alpers GW, Roberts H, Koopman C, Adams RE, Ernst H, Dev P, Taylor CB: Evaluation of an internet support group for women with primary breast cancer. Cancer 2003;97:1164-1173.

26 Hiller W, Goebel G: Rapid assessment of tinnitus-related psychological distress using the Mini-TQ. Int J Audiol 2004;43:600-604.

27 Donker T, van Straten A, Marks I, Cuijpers P: A brief Web-based screening questionnaire for common mental disorders: Development and validation. J Med Internet Res 2009;11:e19.

28 Janca A, Hiller W: ICD-10 checklists -a tool for clinicians' use of the ICD-10 classification of mental and behavioral disorders. Compr Psychiatry 1996;37:180-187. 
29 Kaldo V, Andersson G: Kognitiv beteendeterapi vid tinnitus [Cognitive-behavioural therapy of tinnitus]. Lund, Studentlitteratur, 2004.

30 Dobie RA: A review of randomized clinical trials in tinnitus. Laryngoscope 1999;109:1202-1211.

31 Andersson G, Kaldo-Sandström V, Ström L, Strömgren T: Internet administration of the Hospital Anxiety and Depression Scale in a sample of tinnitus patients. J Psychosom Res 2003;55:259-262.

32 Newman CW, Jacobson GP, Spitzer JB: Development of the Tinnitus Handicap Inventory. Arch Otolaryngol Head Neck Surg 1996;122:143-148.

33 Kleinjung T, Fischer B, Langguth B, Sand PG, Hajak G, Dvorakova J, Eichhammer P: Validation of the German version of the Tinnitus Handicap Inventory [Validierung einer deutschsprachigen Version des Tinnitus Handicap Inventory]. Psychiatr Prax 2007;34:140142.

34 Hallam RS: Manual of the Tinnitus Questionnaire (TQ). London, Psychological Corporation, 1996.

35 Zigmond AS, Snaith RP: The hospital anxiety and depression scale. Acta Psychiatr Scand 1983;67:361-370.

36 Herrmann-Lingen C, Buss U, Snaith RP: Hospital Anxiety and Depression Scale Deutsche Version (HADS-D) (3., aktualisierte und neu normierte Auflage). Manual. Bern, Hans Huber, 2011.

37 Morin CM: Insomnia: psychological assessment and management. New York, Guilford Press, 1993.

38 Pillmann F: The Halle Sleep Interview [Das Hallenser Schlafinterview], 2004, 39 Westin V, Hayes SC, Andersson G: Is it the sound or your relationship to it? The role of acceptance in predicting tinnitus impact. Behav Res Ther 2008;46:1259-1265. 
40 Weise C, Kleinstäuber M, Hesser H, Westin VZ, Andersson G: Acceptance of tinnitus: validation of the tinnitus acceptance questionnaire. Cogn Behav Ther 2013;42:100-115. 41 Devilly GJ, Borkovec TD: Psychometric properties of the credibility/expectancy questionnaire. J Behav Ther Exp Psychiatry 2000;31:73-86.

42 Schafer JL: Analysis of incomplete multivariate data. New York, Chapman \& Hall, 2000.

43 Jacobson NS, Truax P: Clinical significance: a statistical approach to defining meaningful change in psychotherapy research. J Consult Clin Psychol 1991;59:12-19. 44 Nyenhuis N, Golm D, Kröner-Herwig B: A systematic review and meta-analysis on the efficacy of self-help interventions in tinnitus. Cogn Behav Ther 2013;42:159-169. 45 Hedman E, Andersson G, Ljótsson B, Andersson E, Rück C, Mortberg E, Lindefors N: Internet-based cognitive behavior therapy vs. cognitive behavioral group therapy for social anxiety disorder: A randomized controlled non-inferiority trial. PLoS ONE 2011;6:e18001. 46 Ljótsson B, Hedman E, Andersson E, Hesser H, Lindfors P, Hursti T, Rydh S, Ruck C, Lindefors N, Andersson G: Internet-delivered exposure-based treatment vs. stress management for irritable bowel syndrome: A randomized trial. Am J Gastroenterol 2011;106:1481-1491.

47 Spek V, Nyklicek I, Smits N, Cuijpers P, Riper H, Keyzer J, Pop V: Internet-based cognitive behavioural therapy for subthreshold depression in people over 50 years old: A randomized controlled clinical trial. Psychol Med 2007;37:1797-1806.

48 Couper MP, Kapteyn A, Schonlau M, Winter J: Noncoverage and nonresponse in an Internet survey. Soc Sci Res 2007;36:131-148.

49 Gosling SD, Vazire S, Srivastava S, John OP: Should we trust web-based studies? A comparative analysis of six preconceptions about internet questionnaires. Am Psychol 2004;59:93-104. 
50 Donkin L, Hickie IB, Christensen H, Naismith SL, Neal B, Cockayne NL, Glozier N: Sampling bias in an internet treatment trial for depression. Transl Psychiatry 2012;2:e174.

51 Cosco TD, Doyle F, Ward M, McGee H: Latent structure of the Hospital Anxiety And Depression Scale: A 10-year systematic review. J Psychosom Res 2012;72:180-184.

52 Coyne JC, van Sonderen E: No further research needed: abandoning the Hospital and Anxiety Depression Scale (HADS). J Psychosom Res 2012;72:173-174.

53 Derogatis LR, Melisaratos N: The Brief Symptom Inventory: an introductory report. Psychol Med 1983;13:595-605.

54 Malouff JM, Schutte NS, Zucker LA: Tinnitus-related distress: A review of recent findings. Curr Psychiatry Rep 2011;13:31-36.

55 Zirke N, Seydel C, Szczepek AJ, Olze H, Haupt H, Mazurek B: Psychological comorbidity in patients with chronic tinnitus: analysis and comparison with chronic pain, asthma or atopic dermatitis patients. Qual Life Res 2013;22:263-272.

56 MacKenzie KR: Time-managed group psychotherapy: effective clinical applications. Washington, DC, American Psychiatric Press, 1997.

57 Kivlighan DM, Jr.,, Kivlighan MC: Counselor Intentions in Individual and Group Treatment. J Couns Psychol 2004;51:347-353.

58 Holmes SE, Kivlighan D. M. J: Comparison of therapeutic factors in group and individual treatment processes. J Couns Psychol 2000;47:478-484.

59 McRoberts C, Burlingame GM, Hoag MJ: Comparative efficacy of individual and group psychotherapy: A meta-analytic perspective. Group Dyn- Theor Res 1998;2:101-117. 60 Hiller W, Goebel G: Assessing audiological, pathophysiological, and psychological variables in chronic tinnitus: A study of reliability and search for prognostic factors. Int $\mathbf{J}$ Behav Med 1999;6:312-330.

61 Hiller W, Goebel G, Schindelmann U: Developing a structured interview to assess audiological, aetiological and psychological variables of tinnitus; in Hazell JWP (ed) 
Proceedings of the sixth international tinnitus seminar London, The Tinnitus and Hyperacusis Centre, 1999, pp 277-282.

62 Landgrebe M, Azevedo A, Baguley D, Bauer C, Cacace A, Coelho C, Dornhoffer J, Figueiredo R, Flor H, Hajak G, van de Heyning P, Hiller W, Khedr E, Kleinjung T, Koller M, Lainez JM, Londero A, Martin WH, Mennemeier M, Piccirillo J, De Ridder D, Rupprecht R, Searchfield G, Vanneste S, Zeman F, Langguth B: Methodological aspects of clinical trials in tinnitus: a proposal for an international standard. J Psychosom Res 2012;73:112-121.

63 Marx RG, Bombardier C, Hogg-Johnson S, Wright JG: Clinimetric and psychometric strategies for development of a health measurement scale. J Clin Epidemiol 1999;52:105-111. 64 Tomba E, Bech P: Clinimetrics and clinical psychometrics: macro- and microanalysis. Psychother Psychosom 2012;81:333-343.

65 Kaldo V, Haak T, Buhrman M, Alfonsson S, Larsen HC, Andersson G: Internet-based cognitive behaviour therapy for tinnitus patients delivered in a regular clinical setting: Outcome and analysis of treatment dropout. Cogn Behav Ther 2013;42:146-158. 66 Cima RF, Maes IH, Joore MA, Scheyen DJ, El Refaie A, Baguley DM, Anteunis LJ, van Breukelen GJ, Vlaeyen JW: Specialised treatment based on cognitive behaviour therapy versus usual care for tinnitus: A randomised controlled trial. Lancet 2012;379:1951-1959. 67 Haaga DA: Introduction to the special section on stepped care models in psychotherapy. J Consult Clin Psychol 2000;68:547-548. 
Table 1

Overview of the Internet-based Cognitive Behavior Therapy (ICBT)

\begin{tabular}{|c|c|c|}
\hline Module & Topic & Goals \\
\hline $1-6$ & Applied relaxation & $\begin{array}{l}\text { Tension and relaxation of different muscle groups } \\
\text { Relaxation through controlled breathing } \\
\text { Establishing a relaxation routine in everyday life }\end{array}$ \\
\hline 7 & Positive imagery & Focusing mentally on a positive image to relax \\
\hline 8 & Focus exercises & $\begin{array}{l}\text { Enhancing the ability to shift focus between tinnitus } \\
\text { and other stimuli }\end{array}$ \\
\hline 9 & Exposure to tinnitus & $\begin{array}{l}\text { Reducing negative emotions and avoidance through } \\
\text { exposure and habituation }\end{array}$ \\
\hline $10-11$ & Cognitive restructuring & Identification and restructuring of negative thoughts \\
\hline 12 & Avoidance behavior & $\begin{array}{l}\text { Reducing avoidance behavior, encouraging positive } \\
\text { activities }\end{array}$ \\
\hline $13-18$ & Optional Modules & $\begin{array}{l}\text { i.e., sound enrichment, tinnitus reframing, noise } \\
\text { sensitivity, sleep management, concentration } \\
\text { management, hearing tactics }\end{array}$ \\
\hline
\end{tabular}


Table 2

Overview of the Cognitive Behavior Group Therapy (GCBT)

\begin{tabular}{|c|c|c|}
\hline Session & Topic & Goals \\
\hline 1 & Warming up \& general information & Discussion of expectations and goals \\
\hline $2-7$ & Progressive Muscle Relaxation & $\begin{array}{l}\text { Long version of PMR } \\
\text { Brief version of PMR } \\
\text { 2-min version of PMR }\end{array}$ \\
\hline 2,3 & Psychoeducation 1 & $\begin{array}{l}\text { Information about epidemiology, causes and } \\
\text { mechanism of tinnitus distress }\end{array}$ \\
\hline 4,5 & Cognitive restructuring & $\begin{array}{l}\text { Identification and restructuring of negative } \\
\text { thoughts }\end{array}$ \\
\hline 6 & Focus exercises & $\begin{array}{l}\text { Enhancing the ability to shift focus between } \\
\text { tinnitus and other stimuli }\end{array}$ \\
\hline 7,8 & Avoidance behavior & $\begin{array}{l}\text { Identification and reduction of avoidance } \\
\text { behaviors }\end{array}$ \\
\hline 9 & Patient-doctor communication & Reduction of "doctor-shopping" \\
\hline 10 & Relapse prevention & $\begin{array}{l}\text { Summary and planning how to maintain } \\
\text { therapy success and cope with a relapse }\end{array}$ \\
\hline 10 & Optional topics & $\begin{array}{l}\text { i.e., positive imagery, stress management, } \\
\text { sleep management, hearing tactics }\end{array}$ \\
\hline
\end{tabular}

Note $. \mathrm{PMR}=$ progressive muscle relaxation. 
Table 3

Baseline Demographics and Clinical Characteristics by Intervention Groups

\begin{tabular}{|c|c|c|c|c|}
\hline & $\operatorname{ICBT}(n=41)$ & $\operatorname{GCBT}(n=43)$ & $\mathrm{DF}(n=44)$ & Test statistics \\
\hline Age (years): $M(S D)$ & $51.3(9.8)$ & $50.2(13.1)$ & $52.1(9.0)$ & $F(2,125)=0.33, p=.723$ \\
\hline Gender: $n$ ( $\%$ female $)$ & $16(39.0)$ & $19(44.2)$ & $16(36.4)$ & $\chi^{2}(2, N=128)=0.57, p=.751$ \\
\hline \multicolumn{5}{|l|}{ Occupational status: $n(\%)$} \\
\hline Employed & $32(78.0)$ & $28(65.1)$ & $35(79.5)$ & $\chi^{2}(2, N=128)=2.83, p=.243$ \\
\hline Unemployed, retired & $9(22.0)$ & $15(34.9)$ & $9(20.5)$ & \\
\hline Educational status: $n(\%)$ & & & & $\chi^{2}(4, N=128)=1.51, p=.825$ \\
\hline Primary or secondary school & $14(34.1)$ & $17(39.5)$ & $18(40.9)$ & \\
\hline Qualification for university entrance & $8(19.5)$ & $8(18.6)$ & $5(11.4)$ & \\
\hline Academic degree & $19(46.3)$ & $18(41.9)$ & $21(47.7)$ & \\
\hline Tinnitus duration (years): $M(S D)$ & $9.2(7.9)$ & $8.4(6.9)$ & $8.0(7.1)$ & $F(2,125)=0.35, p=.708$ \\
\hline Tinnitus loudness: $n(\%)$ & & & & $\chi^{2}(4, N=128)=1.53, p=.822$ \\
\hline Only noticeable during silence & $3(7.3)$ & $2(4.7)$ & $2(4.5)$ & \\
\hline
\end{tabular}


Maskable by usual ambient noise $22(53.7)$

Always noticeable, not maskable

$16(39.0)$

$27(62.8)$

$29(65.9)$

Associated otological conditions: $n(\%)$

Hearing deficit

Dizziness

Earlier treatments for tinnitus: $n(\%)$

Blood-flow enhancing medication

Orthopedic treatment

$24(58.5)$

$28(68.3)$

$13(31.7)$

$36(87.8)$

$28(68.3)$

$17(41.5)$

$17(41.5)$

Relaxation training

Alternative Medicine

Psychotherapy

$16(39.0)$

$9(22.0)$

$6(14.6)$

$6(14.6)$

$5(12.2)$

$2(4.9)$

14 (32.6)

13 (29.5)

Hyperbaric Oxygen therapy

Noise generator

Hearing aid

Self-help group
$29(67.4)$

$31(72.1)$

$16(37.2)$

$42(97.7)$

$32(74.4)$

19 (44.2)

$17(39.5)$

13 (30.2)

$4(9.3)$

$3(7.0)$

$6(14.0)$

$4(9.3)$

$3(7.0)$
35 (79.5)

$34(77.3)$

$13(29.5)$

39 (88.6)

$33(75.0)$

18 (40.9)

$16(36.4)$

$12(27.3)$

7 (15.9)

$3(6.8)$

7 (15.9)

4 (11.4)

$2(4.5)$ $\chi^{2}(2, N=128)=4.41, p=.110$

$\chi^{2}(2, N=128)=0.87, p=.647$

$\chi^{2}(2, N=128)=0.61, p=.736$

$\chi^{2}(2, N=128)=3.26, p=.196$

$\chi^{2}(2, N=128)=0.58, p=.748$

$\chi^{2}(2, N=128)=0.11, p=.947$

$\chi^{2}(2, N=128)=0.24, p=.888$

$\chi^{2}(2, N=128)=1.44, p=.486$

$\chi^{2}(2, N=128)=2.55, p=.279$

$\chi^{2}(2, N=128)=1.96, p=.370$

$\chi^{2}(2, N=128)=0.07, p=.967$

$\chi^{2}(2, N=128)=0.19, p=.908$

$\chi^{2}(2, N=128)=0.29, p=.865$ 
Running head: INTERNET-BASED VERSUS GROUP THERAPY FOR TINNITUS

Number of earlier tinnitus treatments: $M(S D)$

$2.9(2.3)$

Note. ICBT = Internet-based cognitive behavior therapy; GCBT = cognitive behavior group therapy; DF = discussion forum 
Table 4

Means, Standard Deviations, and Within-Group Effect Sizes for Each Outcome Measure, Study Group and Measurement

\begin{tabular}{|c|c|c|c|c|c|c|c|c|c|c|c|}
\hline \multirow{2}{*}{$\begin{array}{l}\text { Measure and } \\
\text { Condition }\end{array}$} & \multirow[b]{2}{*}{$n$} & \multicolumn{2}{|c|}{ Pre } & \multicolumn{2}{|c|}{ Post } & \multicolumn{2}{|c|}{$6 \mathrm{mo} \mathrm{FU}$} & \multicolumn{4}{|c|}{ Cohen's $d[95 \%-\mathrm{CI}]$} \\
\hline & & $M$ & $S D$ & $M$ & $S D$ & $M$ & $S D$ & & $e-$ Post & & $6 \mathrm{mo} \mathrm{FU}$ \\
\hline \multicolumn{12}{|l|}{ Mini-TQ } \\
\hline ICBT & 41 & 12.20 & 4.58 & 7.44 & 5.30 & 6.62 & 6.08 & 0.96 & {$[0.48,1.44]$} & 1.03 & {$[0.55,1.52]$} \\
\hline GCBT & 43 & 14.19 & 4.51 & 8.09 & 4.93 & 7.41 & 4.43 & 1.29 & {$[0.79,1.79]$} & 1.52 & {$[0.99,2.05]$} \\
\hline $\mathrm{DF}$ & 44 & 12.50 & 4.83 & 11.09 & 5.77 & & & 0.26 & {$[-0.16,0.69]$} & & \\
\hline \multicolumn{12}{|l|}{ THI } \\
\hline ICBT & 41 & 40.34 & 17.64 & 26.67 & 20.75 & 24.56 & 34.09 & 0.71 & {$[0.25,1.17]$} & 0.58 & {$[0.13,1.03]$} \\
\hline GCBT & 43 & 44.33 & 19.17 & 27.70 & 21.93 & 26.96 & 21.79 & 0.81 & {$[0.35,1.26]$} & 0.85 & {$[0.39,1.31]$} \\
\hline $\mathrm{DF}$ & 44 & 40.23 & 20.54 & 37.46 & 18.94 & & & 0.14 & {$[-0.28,0.56]$} & & \\
\hline \multicolumn{12}{|l|}{ HADS-A } \\
\hline ICBT & 41 & 7.41 & 3.56 & 5.44 & 3.23 & 5.49 & 4.43 & 0.58 & {$[0.13,1.03]$} & 0.48 & {$[0.03,0.92]$} \\
\hline GCBT & 43 & 7.79 & 3.73 & 5.84 & 3.82 & 5.73 & 3.85 & 0.52 & {$[0.08,0.95]$} & 0.54 & {$[0.11,0.98]$} \\
\hline DF & 44 & 8.00 & 4.24 & 7.67 & 4.68 & & & 0.07 & {$[-0.35,0.49]$} & & \\
\hline
\end{tabular}

HADS-D 
Running head: INTERNET-BASED VERSUS GROUP THERAPY FOR TINNITUS

$\begin{array}{llllllllllll}\text { ICBT } & 41 & 5.95 & 4.21 & 4.41 & 3.72 & 4.00 & 4.30 & 0.39 & {[-0.05,0.83]} & 0.46 & {[0.01,0.90]} \\ \text { GCBT } & 43 & 6.02 & 3.79 & 4.41 & 3.92 & 4.96 & 4.74 & 0.42 & {[-0.01,0.85]} & 0.25 & {[-0.18,0.67]} \\ \text { DF } & 44 & 6.43 & 4.48 & 5.88 & 4.41 & & & 0.13 & {[-0.29,0.55]} & \end{array}$

ISI

$\begin{array}{lccccccccccc}\text { ICBT } & 41 & 12.68 & 5.91 & 8.70 & 5.80 & 8.72 & 6.61 & 0.68 & {[0.22,1.14]} & 0.63 & {[0.18,1.09]} \\ \text { GCBT } & 43 & 12.40 & 6.08 & 9.03 & 6.75 & 8.66 & 5.52 & 0.52 & {[0.09,0.96]} & 0.64 & {[0.20,1.09]} \\ \text { DF } & 44 & 11.25 & 6.51 & 10.91 & 7.21 & & & -0.02 & {[-0.43,0.40]} & \\ \text { TAQ } & & & & & & & & & & & \\ \text { ICBT } & 41 & 42.07 & 11.70 & 47.91 & 11.70 & 49.14 & 12.04 & 0.52 & {[0.07,0.96]} & 0.59 & {[0.14,1.05]} \\ \text { GCBT } & 43 & 40.26 & 11.87 & 46.31 & 11.96 & 48.24 & 13.07 & 0.51 & {[0.07,0.94]} & 0.64 & {[0.20,1.08]} \\ \text { DF } & 44 & 42.84 & 13.48 & 43.99 & 13.51 & & & 0.09 & {[-0.33,0.50]} & \end{array}$

Note ICBT $=$ Internet-based cognitive behavior therapy; GCBT = cognitive behavior group therapy; $\mathrm{DF}=$ discussion forum; Mini-TQ $=$ Mini Tinnitus Questionnaire; THI = Tinnitus Handicap Inventory; HADS-A = Hospital Anxiety and Depression Scale subscale anxiety; HADS-D = Hospital Anxiety and Depression Scale subscale depression; ISI = Insomnia Severity Index; TAQ = Tinnitus Acceptance Questionnaire; 6 mo $\mathrm{FU}=6$-month follow up. 
Table 5

Test Statistics of ANOVAs and Hedges' $g$ for Each Measure

Pre-treatment to 6

\section{Pre-to Post- treatment}

\section{month FU}

\begin{tabular}{llccc}
\cline { 3 - 4 } Comparisons & Measure & Interaction & \\
& & \multicolumn{2}{c}{ time x group } & Hedges' $g$ [95\%-CI] \\
\hline ICBT vs. DF & & & & \\
& & & & \\
& TQ & $F(1,83)=15.01, p<.001$ & 0.65 & {$[0.21,1.09]$} \\
& THI & $F(1,83)=12.12, p=.001$ & 0.56 & {$[0.12,0.99]$} \\
& HADS-A & $F(1,83)=6.51, p=.013$ & 0.41 & {$[-0.02,0.84]$} \\
& HADS-D & $F(1,83)=2.68, p=.105$ & 0.24 & {$[-0.19,0.66]$} \\
& ISI & $F(1,83)=22.55, p<.001$ & 0.64 & {$[0.20,1.07]$} \\
& TAQ & $F(1,82)=4.27, p=.042$ & 0.38 & {$[-0.05,0.80]$}
\end{tabular}

GCBT vs. DF

$\begin{array}{llll}\text { TQ } & F(1,85)=26.78, p<.001 & 0.93 & {[0.49,1.37]} \\ \text { THI } & F(1,85)=17.75, p<.001 & 0.69 & {[0.25,1.12]} \\ \text { HADS-A } & F(1,85)=6.69, p=.011 & 0.39 & {[-0.03,0.82]}\end{array}$




$\begin{array}{llll}\text { HADS-D } & F(1,85)=3.18, p=.078 & 0.26 & {[-0.16,0.68]} \\ \text { ISI } & F(1,85)=12.97, p=.001 & 0.52 & {[0.10,0.95]} \\ \text { TAQ } & F(1,85)=6.69, p=.011 & 0.39 & {[-0.04,0.81]}\end{array}$

ICBT vs. GCBT

$\begin{array}{llllll}\text { TQ } & F(2,164)=1.12, p=.328 & -0.28 & {[-0.71,0.15]} & -0.24 & {[-0.67,0.18]} \\ \text { THI } & F(2,164)=0.25, p=.781 & -0.15 & {[-0.28,0.58]} & -0.07 & {[-0.49,0.36]} \\ \text { HADS-A } & F(2,164)=0.14, p=.866 & 0.01 & {[-0.42,0.44]} & -0.03 & {[-0.46,0.39]} \\ \text { HADS-D } & F(2,164)=1.41, p=.248 & 0.02 & {[-0.41,0.45]} & 0.21 & {[-0.22,0.64]} \\ \text { ISI } & F(2,164)=0.29, p=.747 & 0.10 & {[-0.33,0.53]} & 0.04 & {[-0.39,0.47]} \\ \text { TAQ } & F(2,164)=0.15, p=.865 & -0.02 & {[-0.41,0.45]} & -0.08 & {[-0.50,0.35]}\end{array}$

Note ICBT $=$ Internet-based cognitive behavior therapy; GCBT = cognitive behavior group therapy; $\mathrm{DF}=$ discussion forum; $\mathrm{TQ}=\mathrm{Mini}$ Tinnitus Questionnaire; THI = Tinnitus Handicap Inventory; HADS-A = Hospital Anxiety and Depression Scale, subscale anxiety; HADS-D = Hospital Anxiety and Depression Scale, subscale depression; ISI = Insomnia Severity Index; TAQ = Tinnitus Acceptance Questionnaire; FU = follow up. 


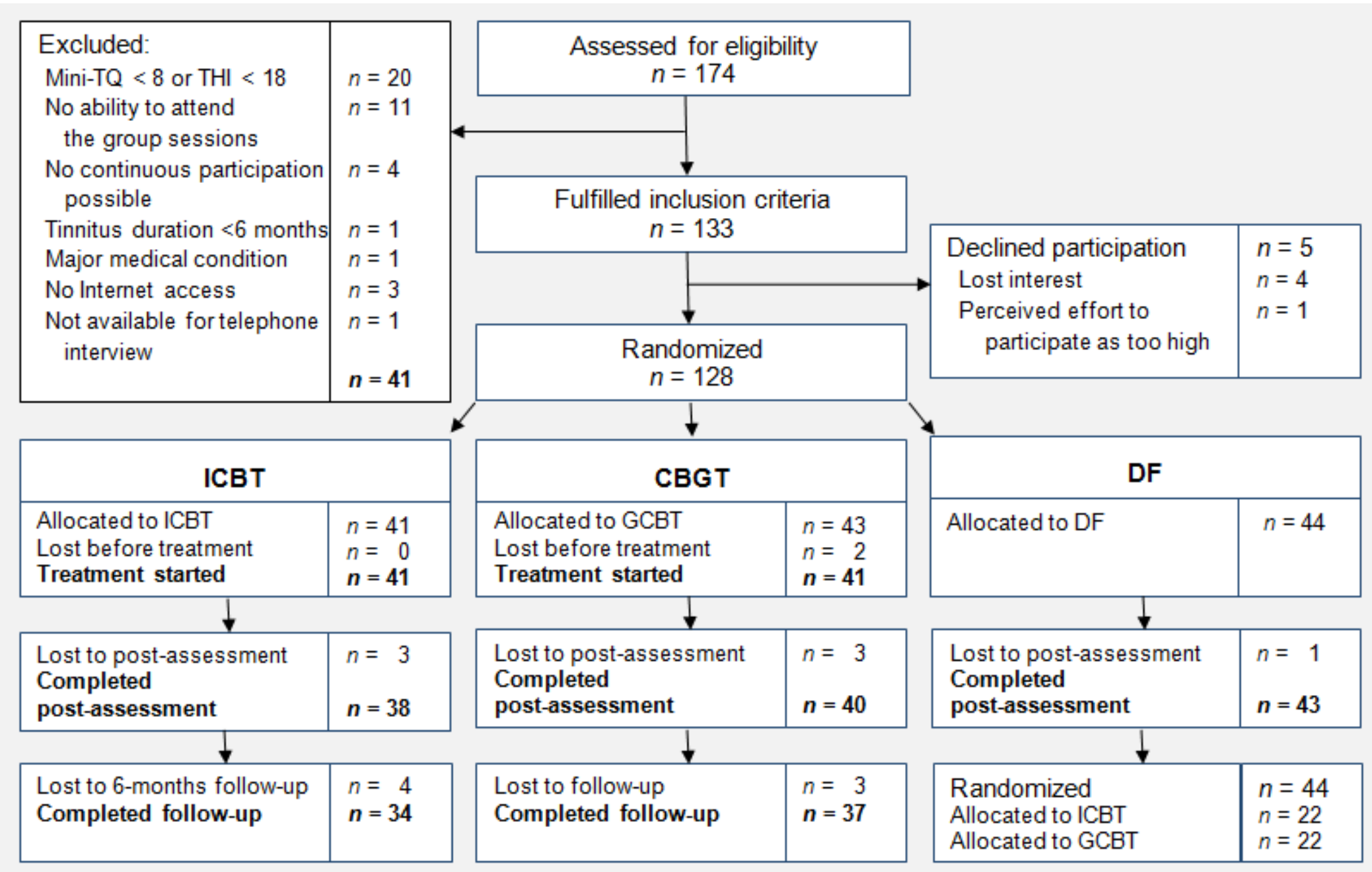

Figure 1. Flowchart of participants through the study (CONSORT diagram). ICBT = Internet-based cognitive behavior therapy; GCBT = cognitive behavior group therapy; $\mathrm{DF}=$ discussion forum. 